\title{
Directional Beamforming for Millimeter-Wave MIMO Systems
}

\author{
Vasanthan Raghavan, Sundar Subramanian, Juergen Cezanne, and Ashwin \\ Sampath
}

\begin{abstract}
The focus of this paper is on beamforming in a millimeter-wave (mmW) multi-input multi-output (MIMO) setup that has gained increasing traction in meeting the high data-rate requirements of nextgeneration wireless systems. For a given MIMO channel matrix, the optimality of beamforming with the dominant right-singular vector (RSV) at the transmit end and with the matched filter to the RSV at the receive end has been well-understood. When the channel matrix can be accurately captured by a physical (geometric) scattering model across multiple clusters/paths as is the case in mmW MIMO systems, we provide a physical interpretation for this optimal structure: beam steering across the different paths with appropriate power allocation and phase compensation. While such an explicit physical interpretation has not been provided hitherto, practical implementation of such a structure in a mmW system is fraught with considerable difficulties (complexity as well as cost) as it requires the use of per-antenna gain and phase control. This paper characterizes the loss in received SNR with an alternate low-complexity beamforming solution that needs only per-antenna phase control and corresponds to steering the beam to the dominant path at the transmit and receive ends. While the loss in received SNR can be arbitrarily large (theoretically), this loss is minimal in a large fraction of the channel realizations reinforcing the utility of directional beamforming as a good candidate solution for mmW MIMO systems.
\end{abstract}

\section{INTRODUCTION}

The ubiquitous nature of communications made possible by the smart-phone and social media revolutions has meant that the data-rate requirements will continue to grow at an exponential rate. On the other hand, even under the most optimistic assumptions, system resources can continue to scale at best at a linear rate leading to enormous mismatches between supply and demand. Given

The authors are with Qualcomm Flarion Technologies, Inc., Bridgewater, NJ 08807, USA. E-mail: \{vraghava, sundars, jcezanne, asampath@qti.qualcomm.com\}. 
this backdrop, many candidate solutions have been proposed [1]-[3] to mesh into the patchwork that addresses the 1000-X data challenge [4] — an intermediate stepping stone towards bridging this burgeoning gap.

One such solution that has gained increasing traction over the last few years is communications over the millimeter-wave $(\mathrm{mmW})$ regime [5]-[8] where the carrier frequency is in the 30 to $300 \mathrm{GHz}$ range. Spectrum crunch, which is the major bottleneck at lower/cellular carrier frequencies, is less problematic at higher carrier frequencies due to the availability of large (either unlicensed or lightly licensed) bandwidths. However, the high frequency-dependent propagation and shadowing losses (that can offset the link margin substantially) complicate the exploitation of these large bandwidths. It is visualized that these losses can be mitigated by limiting coverage to small areas and leveraging the small wavelengths that allows the deployment of a large number of antennas in a fixed array aperture.

Despite the possibility of multi-input multi-output (MIMO) communications, mmW signaling differs significantly from traditional MIMO architectures at cellular frequencies. The most optimistic antenna configurations 1 at cellular frequencies are on the order of $4 \times 8$ with a precoder rank (number of layers) of 1 to 4; see, e.g., [9]. Higher rank signaling requires multiple radio-frequency (RF) chains 2 which are easier to realize at lower frequencies than at the $\mathrm{mmW}$ regime. Thus, there has been a growing interest in understanding the capabilities of low-complexity approaches such as beamforming (that require only a single RF chain) in mmW systems [10]-[15].

On the other hand, smaller form factors at $\mathrm{mmW}$ frequencies ensure 3 that configurations such as $4 \times 64$ are realistic. Such high antenna dimensionalities as well as the considerably large bandwidths at $\mathrm{mmW}$ frequencies result in a higher resolvability of the multipath and thus, the MIMO channel is naturally sparser in the mmW regime than at cellular frequencies [16]-[18]. In particular, the highly directional nature of the channel ensures the relevance of physicallymotivated beam steering at either end, which is difficult (if not impossible) at cellular frequen-

\footnotetext{
${ }^{1}$ In a downlink setting, the first dimension corresponds to the number of antennas at the user equipment end and the second at the base-station end.

${ }^{2}$ An RF chain includes (but is not limited to) analog-to-digital and digital-to-analog converters, power and low-noise amplifiers, mixers, etc.

${ }^{3}$ For example, a 64 element uniform linear array (ULA) at $30 \mathrm{GHz}$ requires an aperture of $\sim 1$ foot at the critical $\lambda / 2$ spacing — a constraint that can be realized at the base-station end.
} 
cies. While this physical connection has been implicitly and intuitively understood, an explicit characterization of this connection has remained absent so far.

We start with such an explicit physical interpretation in this work by showing that the optimal beamformer structure corresponds to beam steering across the different paths that capture the MIMO channel with appropriate power allocation and phase compensation. We also illustrate the structure of this power allocation and phase compensation in many interesting special cases. Despite using only a single RF chain, the optimal beamformer requires per-antenna phase and gain control (in general), which could render this scheme disadvantageous from a cost perspective. Thus, we study the loss in received SNR with a simpler scheme that requires only phase control and steers beams to the dominant path at either end. Our study shows that this simpler scheme suffers only a minimal loss relative to the optimal beamforming scheme in a large fraction of the channel realizations, thus making it attractive from a practical standpoint. Notations: Lower- $(\mathbf{x})$ and upper-case block $(\mathbf{X})$ letters denote vectors and matrices with $\mathrm{x}(i)$ and $\mathbf{X}(i, j)$ denoting the $i$-t $\mathrm{h}$ and $(i, j)$-th entries of $\mathbf{x}$ and $\mathbf{X}$, respectively. $\|\mathbf{x}\|_{2}$ denotes the 2-norm of a vector $\mathbf{x}$ (that is, $\|\mathbf{x}\|_{2}=\left(\sum_{i}|\mathbf{x}(i)|^{2}\right)^{1 / 2}$ ), whereas $\mathbf{x}^{H}$ and $\mathbf{x}^{T}$ denote the complex conjugate Hermitian and regular transposition operations of $x$, respectively. We use $\mathbb{Z}, \mathbb{R}, \mathbb{R}^{+}$and $\mathbb{C}$ to denote the field of integers, real numbers, positive reals and complex numbers, respectively.

\section{System SETUP}

Let $\mathbf{H}$ denote the $N_{r} \times N_{t}$ channel matrix with $N_{r}$ receive and $N_{t}$ transmit antennas. We assume an extended Saleh-Valenzuela geometric model [19] for the channel where $\mathbf{H}$ is determined by scattering over $L$ clusters 4 and denoted as follows:

$$
\mathbf{H}=\sqrt{\frac{N_{r} N_{t}}{L}} \cdot \sum_{\ell=1}^{L} \alpha_{\ell} \cdot \mathbf{u}_{\ell} \mathbf{v}_{\ell}^{H}
$$

where $\alpha_{\ell} \sim \mathcal{C N}(0,1)$ denotes the complex gain, $\mathbf{u}_{\ell}$ denotes the $N_{r} \times 1$ receive array steering vector, and $\mathbf{v}_{\ell}$ denotes the $N_{t} \times 1$ transmit array steering vector, all corresponding to the $\ell$-th path. With this assumption, the normalization constant $\sqrt{\frac{N_{r} N_{t}}{L}}$ in $\mathbf{H}$ ensures that the standard channel power normalization in MIMO system studies holds. As a typical example of the case where

\footnotetext{
${ }^{4}$ Each cluster is assumed to have one dominant path and diffuse scattering over a cluster with multiple sub-paths is not captured here.
} 
a uniform linear array (ULA) of antennas are deployed at both ends of the link (and without loss of generality pointing along the $X$ axis), the array steering vectors $\mathbf{u}_{\ell}$ and $\mathbf{v}_{\ell}$ corresponding to angle of arrival (AoA) $\phi_{\mathrm{R}, \ell}$ and angle of departure (AoD) $\phi_{\mathrm{T}, \ell}$ in the azimuth (assuming an elevation angle $\theta_{\mathrm{R}, \ell}=\theta_{\mathrm{T}, \ell}=90^{\circ}$ ) are given as

$$
\begin{aligned}
& \mathbf{u}_{\ell}=\frac{1}{\sqrt{N_{r}}} \cdot\left[1 e^{j k d_{\mathrm{R}} \cos \left(\phi_{\mathrm{R}, \ell}\right)} e^{j 2 k d_{\mathrm{R}} \cos \left(\phi_{\mathrm{R}, \ell}\right)} \cdots e^{j\left(N_{r}-1\right) k d_{\mathrm{R}} \cos \left(\phi_{\mathrm{R}, \ell}\right)}\right]^{T} \\
& \mathbf{v}_{\ell}=\frac{1}{\sqrt{N_{t}}} \cdot\left[1 e^{j k d_{\mathrm{T}} \cos \left(\phi_{\mathrm{T}, \ell}\right)} e^{j 2 k d_{\mathrm{T}} \cos \left(\phi_{\mathrm{T}, \ell}\right)} \cdots e^{j\left(N_{t}-1\right) k_{\mathrm{T}} d \cos \left(\phi_{\mathrm{T}, \ell}\right)}\right]^{T}
\end{aligned}
$$

where $k=\frac{2 \pi}{\lambda}$ is the wave number with $\lambda$ the wavelength of propagation, and $d_{\mathrm{R}}$ and $d_{\mathrm{T}}$ are the inter-antenna element spacing at the receiver and transmitter sides, respectively. To simplify the notations and to capture the constant phase offset (CPO)-nature of the array-steering vectors and the correspondence with their respective physical angles, we will henceforth 5 denote $\mathbf{u}_{\ell}$ and $\mathbf{v}_{\ell}$ in (2)-(3) as $\operatorname{CPO}\left(\phi_{\mathrm{R}, \ell}\right)$ and $\operatorname{CPO}\left(\phi_{\mathrm{T}, \ell}\right)$, respectively. With the typical $d_{\mathrm{R}}=d_{\mathrm{T}}=\frac{\lambda}{2}$ spacing, we have $k d_{\mathrm{R}}=k d_{\mathrm{T}}=\pi$. In the general case where the paths depart at an AoD pair of $\left(\theta_{\mathrm{T}, \ell}, \phi_{\mathrm{T}, \ell}\right)$ and arrive at an AoA pair of $\left(\theta_{\mathrm{R}, \ell}, \phi_{\mathrm{R}, \ell}\right)$ in the elevation and azimuth, respectively, the $\cos \left(\phi_{\mathrm{R}, \ell}\right)$ and $\cos \left(\phi_{\mathrm{T}, \ell}\right)$ terms in (2)-(3) are replaced with $\sin \left(\theta_{\mathrm{R}, \ell}\right) \cos \left(\phi_{\mathrm{R}, \ell}\right)$ and $\sin \left(\theta_{\mathrm{T}, \ell}\right) \cos \left(\phi_{\mathrm{T}, \ell}\right)$, respectively. Similar expressions for $\mathbf{u}_{\ell}$ and $\mathbf{v}_{\ell}$ can be written if the array is placed on the $\mathrm{Y}$ or $\mathrm{Z}$ axes or with a planar array; see [13], [20], for example.

We are interested in beamforming (rank-1 signaling) over $\mathbf{H}$ with the unit-norm $N_{t} \times 1$ beamforming vector $\mathbf{f}$. The system model in this setting is given as

$$
\mathbf{y}=\sqrt{\rho_{\text {prebf }}} \cdot \mathbf{H f} s+\mathbf{n}
$$

where $\rho_{\text {prebf }}$ is the pre-beamforming SNR, $s$ is the symbol chosen from an appropriate constellation for signaling, and $\mathbf{n}$ is the $N_{r} \times 1$ proper complex white Gaussian noise vector (that is, $\mathbf{n} \sim \mathcal{C N}(\mathbf{0}, \mathbf{I}))$ added at the receiver. The symbol $s$ is decoded by beamforming at the receiver along the unit-norm $N_{r} \times 1$ vector $\mathrm{g}$ to obtain

$$
\widehat{s}=\mathbf{g}^{H} \mathbf{y}=\sqrt{\rho_{\text {prebf }}} \cdot \mathbf{g}^{H} \mathbf{H} \mathbf{f} s+\mathbf{g}^{H} \mathbf{n} .
$$

Let $\mathcal{F}_{2}$ denote the class of energy-constrained beamforming vectors. That is, $\mathcal{F}_{2}=\left\{\mathbf{f}:\|\mathbf{f}\|_{2} \leq\right.$ 1 . Under perfect channel state information (CSI) (that is, $\mathbf{H}=\mathbf{H}$ ) at both the transmitter and

\footnotetext{
${ }^{5}$ Similar notation will also be followed for other vectors with a constant phase offset across the array.
} 
the receiver, optimal beamforming vectors $\mathbf{f}_{\text {opt }}$ and $\mathbf{g}_{\text {opt }}$ are to be designed from $\mathcal{F}_{2}$ to maximize the received SNR [21], defined as,

$$
\mathrm{SNR}_{\mathrm{rx}} \triangleq \rho_{\text {prebf }} \cdot \frac{\left|\mathbf{g}^{H} \mathbf{H} \mathbf{f}\right|^{2} \cdot \mathbf{E}\left[|s|^{2}\right]}{\mathbf{E}\left[\left|\mathbf{g}^{H} i \mathbf{n}\right|^{2}\right]}=\rho_{\text {prebf }} \cdot \frac{\left|\mathbf{g}^{H} \mathbf{H} \mathbf{f}\right|^{2}}{\mathbf{g}^{H} \mathbf{g}}
$$

Clearly, the above quantity is maximized with $\left\|\mathbf{f}_{\mathrm{opt}}\right\|_{2}=1$, otherwise energy is unused in beamforming. Further, a simple application of Cauchy-Schwarz inequality shows that $\mathrm{g}_{\mathrm{opt}}$ is a matched filter combiner at the receiver with $\left\|\mathbf{g}_{\text {opt }}\right\|_{2}=1$ resulting in $\operatorname{SNR}_{\mathrm{rx}}=\rho_{\text {prebf }} \cdot \mathbf{f}^{H} \mathrm{H}^{H} \mathbf{H} \mathbf{f}$. We thus have

$$
\mathbf{f}_{\mathrm{opt}}=\mathrm{v}_{1}\left(\mathrm{H}^{H} \mathrm{H}\right), \quad \mathbf{g}_{\mathrm{opt}}=\frac{H \mathrm{v}_{1}\left(\mathrm{H}^{H} \mathrm{H}\right)}{\left\|H \mathrm{v}_{1}\left(\mathrm{H}^{H} \mathrm{H}\right)\right\|_{2}},
$$

where $v_{1}\left(\mathrm{H}^{H} \mathrm{H}\right)$ denotes a dominant unit-norm right singular vector of $\mathrm{H}$. Here, the singular value decomposition of $\mathrm{H}$ is given as $\mathrm{H}=\mathrm{U} \wedge \mathrm{V}^{H}$ with $\mathrm{U}$ and $\mathrm{V}$ being $N_{r} \times N_{r}$ and $N_{t} \times N_{t}$ unitary matrices of left and right singular vectors, respectively, and arranged so that the corresponding leading diagonal entries of the $N_{r} \times N_{t}$ singular value matrix $\Lambda$ are in non-increasing order.

\section{EXPLICIT CONNECTION BETWEEN $\mathbf{f}_{\text {opt }}$, $\mathbf{g}_{\text {opt }}$ AND PHYSICAL DIRECTIONS}

A typical sparse mmW channel can be assumed to consist of a small number of dominant clusters (say, $L=2$ or 3) [7], [16]-[18], [22]. For example, a dominant line-of-sight (LOS) path with strong reflectors in the form of a few glass windows of buildings in the vicinity of the transmitter or the receiver could capture an urban mmW setup. In the context of such a sparse mmW channel $H$, the intuitive meaning of $\mathbf{f}_{\mathrm{opt}}$ is to "coherently combine" (by appropriate phase compensation) the energy across the multiple paths so as to maximize the energy delivered to the receiver. The precise connection between the physical directions $\left\{\phi_{\mathrm{R}, \ell}, \phi_{\mathrm{T}, \ell}\right\}$ in the ULA channel model and $f_{o p t}$ in (6) is established next. Towards this goal, a preliminary result is established first.

Proposition 1. With $\mathrm{H}=\mathrm{H}$ and the channel model in (1), all the eigenvectors of $\mathrm{H}^{H} \mathrm{H}$ can be represented as linear combinations of $\mathbf{v}_{1}, \cdots, \mathbf{v}_{L}$.

Proof. See Appendix A

It is important to note that while the right singular vectors of $\mathrm{H}$ (also, the eigenvectoirs of $\mathrm{H}^{H} \mathrm{H}$ ) are orthonormal by construction, $\mathbf{v}_{1}, \cdots, \mathbf{v}_{L}$ need not be orthonormal. With this background, 
Prop. 1 provides a non-unitary basis for the eigen-space of $\mathrm{H}^{H} \mathrm{H}$ when $L \leq N_{t}$. As another ramification of this fact, in the case where $L>N_{t}$, it is still true that the set $\left\{\mathbf{v}_{1}, \cdots, \mathbf{v}_{L}\right\}$ spans the eigen-space of $\mathrm{H}^{H} \mathrm{H}$, however this set is no longer a basis. These facts along with the fact that $\mathbf{f}_{\text {opt }}$ is a dominant eigenvector of $\mathrm{H}^{H} \mathrm{H}$ also implies the following:

$$
\frac{\mathrm{SNR}_{\mathrm{rx}}}{\rho_{\text {prebf }}}=\max _{\mathbf{f}:\|\mathbf{f}\|_{2}=1} \frac{\mathbf{f}^{H} \mathrm{H}^{H} \mathbf{H} \mathbf{f}}{\mathbf{f}^{H} \mathbf{f}}=\max _{\mathbf{f}: \mathbf{f} \in \mathcal{G}_{0}} \frac{\mathbf{f}^{H} \mathrm{H}^{H} \mathbf{H} \mathbf{f}}{\mathbf{f}^{H} \mathbf{f}}
$$

where $\mathcal{G}_{0} \triangleq\left\{\mathbf{f}: \mathbf{f}=\sum_{i=1}^{L} e^{j \theta_{i}} \beta_{i} \mathbf{v}_{i}\right\}$ with $\beta_{i} \in \mathbb{R}^{+}, \theta_{i} \in[0,2 \pi), i=1, \cdots, L$. Without loss in generality, we can set $\beta_{L}=\sqrt{1-\sum_{j=1}^{L-1} \beta_{j}^{2}}$ and $\theta_{1}=0$ in the definition of $\mathcal{G}_{0}$ to reduce the optimization in (7) to a $2(L-1)$-dimensional optimization over $\mathcal{G}$, defined as,

$$
\mathcal{G} \triangleq\left\{\mathbf{f}: \mathbf{f}=\beta_{1} \mathbf{v}_{1}+\sum_{i=2}^{L-1} e^{j \theta_{i}} \beta_{i} \mathbf{v}_{i}+e^{j \theta_{L}} \sqrt{1-\sum_{j=1}^{L-1} \beta_{j}^{2} \mathbf{v}_{L}}\right\}
$$

In other words, the optimization over the space of $\mathcal{G}$ should result in the dominant right singular vector of $\mathrm{H}^{H} \mathrm{H}$. Note that the constraint set in the optimization over $\mathcal{G}$ is the outer product of a $(L-1)$-dimensional real sphere where $\sum_{i=1}^{L-1} \beta_{i}^{2} \leq 1$ with a cuboid $\prod_{i=2}^{L} \theta_{i}, 0 \leq \theta_{i}<2 \pi$.

We now consider the special case where $L=2$ and perform this optimization and thus provide a physical interpretation of $\mathbf{f}_{\mathrm{opt}}$. For this, note that in the $L=2$ case, $\mathrm{H}^{H} \mathrm{H}$ simplifies to

$$
\frac{L}{N_{t} N_{r}} \cdot \mathbf{H}^{H} \mathbf{H}=\left|\alpha_{1}\right|^{2} \cdot \mathbf{v}_{1} \mathbf{v}_{1}^{H}+\left|\alpha_{2}\right|^{2} \cdot \mathbf{v}_{2} \mathbf{v}_{2}^{H}+\alpha_{1}^{\star} \alpha_{2} \cdot\left(\mathbf{u}_{1}^{H} \mathbf{u}_{2}\right) \cdot \mathbf{v}_{1} \mathbf{v}_{2}^{H}+\alpha_{2}^{\star} \alpha_{1} \cdot\left(\mathbf{u}_{2}^{H} \mathbf{u}_{1}\right) \cdot \mathbf{v}_{2} \mathbf{v}_{1}^{H} .
$$

With $\beta_{1}=\beta$ and $\theta_{2}=\theta$ in the archetypical $\mathbf{f}$ from $\mathcal{G}$, the norm of $\mathbf{f}$ is given as

$$
\mathbf{f}^{H} \mathbf{f}=1+2 \beta \sqrt{1-\beta^{2}} \cdot\left|\mathbf{v}_{1}^{H} \mathbf{v}_{2}\right| \cdot \cos (\phi)
$$

where $\phi \triangleq \theta+\angle \mathbf{v}_{1}^{H} \mathbf{v}_{2}$. Further, a tedious but straightforward calculation shows that

$$
\begin{aligned}
& \frac{L}{N_{t} N_{r}} \cdot \mathbf{f}^{H} \mathbf{H}^{H} \mathbf{H} \mathbf{f} \\
& =\beta^{2}\left|\alpha_{1}\right|^{2}+\left(1-\beta^{2}\right)\left|\alpha_{2}\right|^{2}+\left(\beta^{2}\left|\alpha_{2}\right|^{2}+\left(1-\beta^{2}\right)\left|\alpha_{1}\right|^{2}\right)\left|\mathbf{v}_{1}^{H} \mathbf{v}_{2}\right|^{2} \\
& \quad+2\left|\alpha_{1}\right|\left|\alpha_{2}\right| \cdot\left|\mathbf{v}_{1}^{H} \mathbf{v}_{2}\right| \cdot\left|\mathbf{u}_{1}^{H} \mathbf{u}_{2}\right| \cdot \cos (\nu)+2 \beta \sqrt{1-\beta^{2}} \cdot\left(\left|\alpha_{1}\right|^{2}+\left|\alpha_{2}\right|^{2}\right) \cdot\left|\mathbf{v}_{1}^{H} \mathbf{v}_{2}\right| \cdot \cos (\phi) \\
& \quad+2 \beta \sqrt{1-\beta^{2}} \cdot\left|\alpha_{1}\right|\left|\alpha_{2}\right| \cdot\left|\mathbf{u}_{1}^{H} \mathbf{u}_{2}\right| \cdot\left[\left|\mathbf{v}_{1}^{H} \mathbf{v}_{2}\right|^{2} \cdot \cos (\nu+\phi)+\cos (\nu-\phi)\right]
\end{aligned}
$$

where $\nu \triangleq \angle \mathbf{v}_{1}^{H} \mathbf{v}_{2}-\angle \mathbf{u}_{1}^{H} \mathbf{u}_{2}+\angle \alpha_{1}-\angle \alpha_{2}$. Observe that the phase term $\nu$ captures the phase misalignment between the two paths since $\left|\mathbf{u}_{i}^{H} \mathbf{H} \mathbf{v}_{j}\right|$ is maximized for all $\{i, j\} \in 1,2$ when $\nu=0$ (coherent phase alignment). 
We now consider many special cases to study the performance of the beamforming scheme. For this, we define the normalized received SNR (denoted as $\widetilde{S N R}$ rx ):

$$
\widetilde{\mathrm{SNR}}_{\mathrm{rx}} \triangleq \frac{\mathrm{SNR}_{\mathrm{rx}}}{N_{t} N_{r} \cdot \rho_{\text {prebf }}}=\frac{1}{N_{t} N_{r}} \cdot \frac{\mathbf{f}^{H} \mathrm{H}^{H} \mathrm{Hf}}{\mathbf{f}^{H} \mathbf{f}}
$$

We start with a physical interpretation for the inner product between $\mathbf{u}_{1}$ and $\mathbf{u}_{2}$ (a similar interpretation holds for $\mathbf{v}_{1}^{H} \mathbf{v}_{2}$ ), corresponding to CPO beams in two directions/paths. With the assumption for $\mathbf{u}_{\ell}$ in (2), we have

$$
\mathbf{u}_{1}^{H} \mathbf{u}_{2}=\exp \left(\frac{j \pi\left(N_{r}-1\right) \cdot \Delta \cos \left(\phi_{\mathrm{R}}\right)}{2}\right) \cdot \frac{\sin \left(N_{r} \pi \Delta \cos \left(\phi_{\mathrm{R}}\right) / 2\right)}{N_{r} \sin \left(\pi \Delta \cos \left(\phi_{\mathrm{R}}\right) / 2\right)}
$$

where $\Delta \cos \left(\phi_{\mathrm{R}}\right) \triangleq \cos \left(\phi_{\mathrm{R}, 2}\right)-\cos \left(\phi_{\mathrm{R}, 1}\right)$. Clearly, the maximum magnitude of $\mathbf{u}_{1}^{H} \mathbf{u}_{2}$ is 1 which is achieved when $\Delta \cos \left(\phi_{\mathrm{R}}\right)=0$ (or when the two paths can be coherently combined in the physical angle space). Further, a minimum magnitude of 0 is achieved in (9) when $\Delta \cos \left(\phi_{\mathrm{R}}\right)=$ $\frac{2 n}{N_{r}}, n \in \mathbb{Z} \backslash\{0\}$. We denote this condition as electrical orthogonality between the two paths, which is achievable with higher regularity in the physical angle space as $N_{r}$ increases.

\section{A. Beamforming along the dominant path}

We start with a scheme where the entire power is directed along only one path (the dominant one): either $\mathbf{v}_{1}(\beta=1)$ or $\mathbf{v}_{2}(\beta=0)$. Note that this scheme is amenable to analog $(\mathrm{RF})$ beamforming as it can be implemented with analog phase shifters alone. As a result, this scheme is of low-complexity and is advantageous in mmW MIMO systems. In contrast, $\mathbf{f}_{\text {opt }}$ requires digital beamforming (in general) - a higher complexity implementation — as it requires both phase shifters and gain control stages. Thus, it is important to characterize the performance achievable with beamforming along the dominant path in benchmarking the performance of the optimal scheme.

It is straightforward to see that this scheme results in the following received SNR:

$$
\begin{aligned}
\widetilde{\operatorname{SNR}}_{\mathrm{rx}}=\frac{1}{L} & \cdot \max \left(\left|\alpha_{1}\right|^{2}+\left|\alpha_{2}\right|^{2}\left|\mathbf{v}_{1}^{H} \mathbf{v}_{2}\right|^{2},\left|\alpha_{2}\right|^{2}+\left|\alpha_{1}\right|^{2}\left|\mathbf{v}_{1}^{H} \mathbf{v}_{2}\right|^{2}\right) \\
& +\frac{1}{L} \cdot 2\left|\alpha_{1}\right|\left|\alpha_{2}\right| \cdot\left|\mathbf{v}_{1}^{H} \mathbf{v}_{2}\right| \cdot\left|\mathbf{u}_{1}^{H} \mathbf{u}_{2}\right| \cdot \cos (\nu) .
\end{aligned}
$$

With $\max \left(\left|\alpha_{1}\right|^{2},\left|\alpha_{2}\right|^{2}\right)=K^{2}$ and $\min \left(\left|\alpha_{1}\right|^{2},\left|\alpha_{2}\right|^{2}\right)=1$ where $K \geq 1$, we have

$$
\begin{aligned}
\widetilde{\mathrm{SNR}}_{\mathrm{rx}} & =\frac{1}{L} \cdot\left(K^{2}+\left|\mathbf{v}_{1}^{H} \mathbf{v}_{2}\right|^{2}+2 K \cdot\left|\mathbf{v}_{1}^{H} \mathbf{v}_{2}\right| \cdot\left|\mathbf{u}_{1}^{H} \mathbf{u}_{2}\right| \cdot \cos (\nu)\right) \\
& \stackrel{(a)}{\leq} \frac{1}{L} \cdot\left(K^{2}+\left|\mathbf{v}_{1}^{H} \mathbf{v}_{2}\right|^{2}+2 K \cdot\left|\mathbf{v}_{1}^{H} \mathbf{v}_{2}\right| \cdot\left|\mathbf{u}_{1}^{H} \mathbf{u}_{2}\right|\right)
\end{aligned}
$$


with equality achieved in (a) in the most optimistic scenario of coherent phase alignment $(\nu=0)$. Clearly, the upper bound is increasing in $K,\left|\mathbf{v}_{1}^{H} \mathbf{v}_{2}\right|$ and $\left|\mathbf{u}_{1}^{H} \mathbf{u}_{2}\right|$. Under favorable channel conditions $\left(\left\{\left|\mathbf{v}_{1}^{H} \mathbf{v}_{2}\right|,\left|\mathbf{u}_{1}^{H} \mathbf{u}_{2}\right|\right\} \approx 1\right)$, beamforming along a single path can yield $\frac{(K+1)^{2}}{L}$, corresponding to a case where the two paths coherently add at the receiver to increase the signal amplitude. When only one of the paths is strong $(K \gg 1)$ or when $\mathbf{v}_{1}$ and $\mathbf{v}_{2}$ are electrically orthogonal, this coherent gain is lost and the beamforming gain is $\frac{K^{2}}{L}$.

\section{B. $\mathbf{v}_{1}$ and $\mathbf{v}_{2}$ are orthogonal}

Proposition 2. When $\mathbf{v}_{1}$ and $\mathbf{v}_{2}$ are electrically orthogonal, the non-unit-norm version of $\mathbf{f}_{\mathrm{RSV}}$ is given as

$$
\mathbf{f}_{\mathrm{RSV}}=\beta_{\mathrm{opt}} \mathbf{v}_{1}+e^{j\left(\angle \alpha_{1}-\angle \alpha_{2}-\angle \mathbf{u}_{1}^{H} \mathbf{u}_{2}\right)} \sqrt{1-\beta_{\mathrm{opt}}^{2}} \mathbf{v}_{2}
$$

where

$$
\beta_{\mathrm{opt}}^{2}=\frac{1}{2} \cdot\left[1+\frac{\left|\alpha_{1}\right|^{2}-\left|\alpha_{2}\right|^{2}}{\sqrt{\left(\left|\alpha_{1}\right|^{2}-\left|\alpha_{2}\right|^{2}\right)^{2}+4\left|\alpha_{1}\right|^{2}\left|\alpha_{2}\right|^{2} \cdot\left|\mathbf{u}_{1}^{H} \mathbf{u}_{2}\right|^{2}}}\right] .
$$

The non-unit-norm version of $\mathrm{g}_{\mathrm{opt}}$ satisfies

$$
\mathbf{g}_{\mathrm{opt}}=\alpha_{1} \beta_{\mathrm{opt}} \cdot \mathbf{u}_{1}+e^{j\left(\angle \alpha_{1}-\angle \alpha_{2}-\angle \mathbf{u}_{1}^{H} \mathbf{u}_{2}\right)} \alpha_{2} \sqrt{1-\beta_{\mathrm{opt}}^{2}} \cdot \mathbf{u}_{2} .
$$

Proof. See Appendix B.

While the structure of $\beta_{\mathrm{opt}}^{2}$ is hard to visualize in general, Fig. 11(a) plots it as a function of $\left|\mathbf{u}_{1}^{H} \mathbf{u}_{2}\right|$ for different choices of $K=\frac{\left|\alpha_{1}\right|}{\left|\alpha_{2}\right|}$. From Fig. 1 $(\mathrm{a})$, if $\mathbf{u}_{1}$ and $\mathbf{u}_{2}$ are orthogonal, we see that $\beta_{\mathrm{opt}}$ is either 1 or 0 with full power allocated to the strongest path. In addition, a straightforward calculation shows that

$$
\left|\mathbf{u}_{1}^{H} \mathbf{u}_{2}\right| \rightarrow 1 \Longrightarrow \beta_{\mathrm{opt}}^{2} \rightarrow \frac{\left|\alpha_{1}\right|^{2}}{\left|\alpha_{1}\right|^{2}+\left|\alpha_{2}\right|^{2}}
$$

In terms of loss with respect to beamforming along the dominant path, a simple calculation shows that

$$
\Delta \mathrm{SNR} \triangleq \frac{\left.\widetilde{\mathrm{SNR}_{\mathrm{rx}}}\right|_{\mathrm{RSV}}}{\left.\widetilde{\mathrm{SNR}}_{\mathrm{rx}}\right|_{\text {Dom. path }}}=\frac{\left|\alpha_{1}\right|^{2}+\left|\alpha_{2}\right|^{2}+\sqrt{\left|\alpha_{1}\right|^{4}+\left|\alpha_{2}\right|^{4}+2\left|\alpha_{1}\right|^{2}\left|\alpha_{2}\right|^{2} \cdot\left(2\left|\mathbf{u}_{1}^{H} \mathbf{u}_{2}\right|^{2}-1\right)}}{2 \cdot \max \left(\left|\alpha_{1}\right|^{2},\left|\alpha_{2}\right|^{2}\right)}
$$




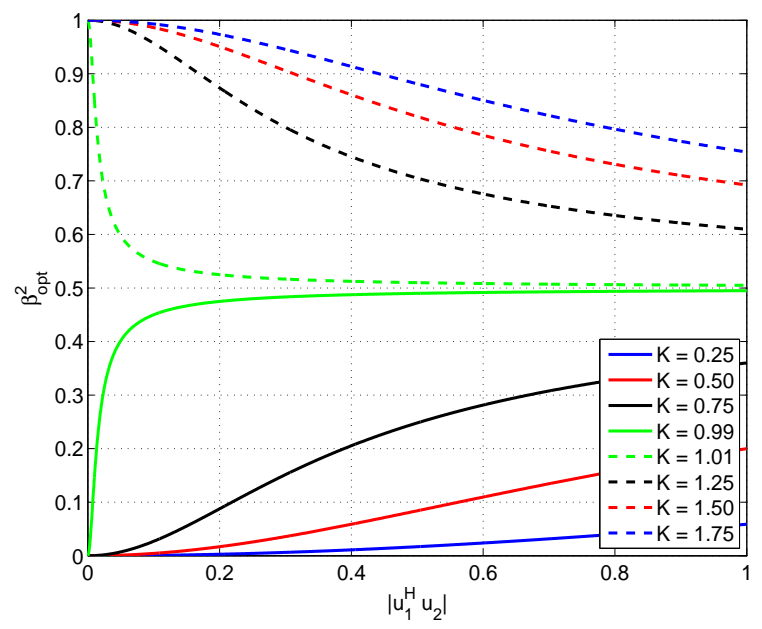

(a)

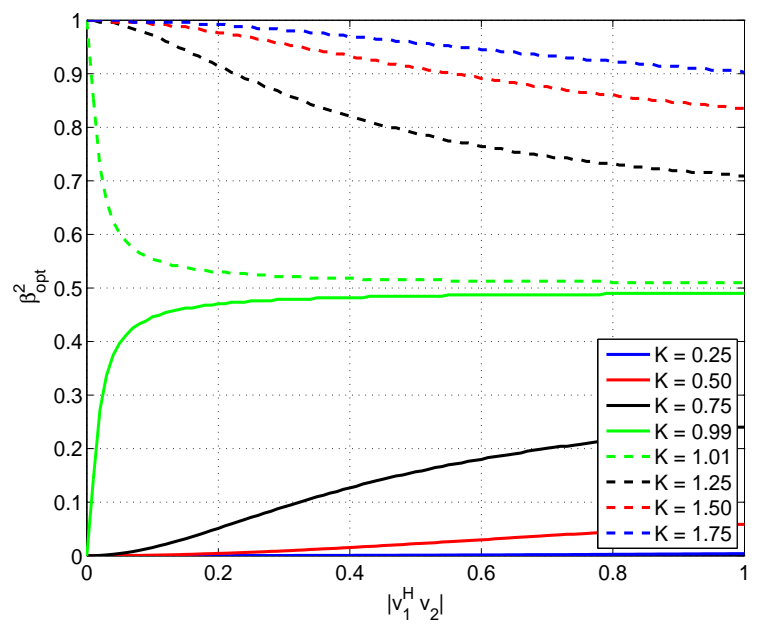

(b)

Fig. 1. $\beta_{\mathrm{opt}}^{2}$ as a function of $K=\frac{\left|\alpha_{1}\right|}{\left|\alpha_{2}\right|}$ for different choices of: a) $\mathbf{u}_{1}$ and $\mathbf{u}_{2}$ when $\mathbf{v}_{1}$ and $\mathbf{v}_{2}$ are orthogonal, and b) $\mathbf{v}_{1}$ and $\mathbf{v}_{2}$ when $\mathbf{u}_{1}$ and $\mathbf{u}_{2}$ are orthogonal.

Clearly, $\triangle$ SNR is increasing in $\left|\mathbf{u}_{1}^{H} \mathbf{u}_{2}\right|$ with

$$
1 \leq \Delta \mathrm{SNR} \leq 1+\frac{\min \left(\left|\alpha_{1}\right|^{2},\left|\alpha_{2}\right|^{2}\right)}{\max \left(\left|\alpha_{1}\right|^{2},\left|\alpha_{2}\right|^{2}\right)}
$$

where the lower bound is realized when $\mathbf{u}_{1}$ and $\mathbf{u}_{2}$ are orthogonal and the upper bound is realized when they are parallel. The above relationship clearly shows that the worst-case performance loss with beamforming along a single path is $3 \mathrm{~dB}$. This SNR loss (in $\mathrm{dB}$ ) is plotted in Fig. 2(a) as a function of $K=\frac{\left|\alpha_{1}\right|}{\left|\alpha_{2}\right|}$ for different choices of $\left|\mathbf{u}_{1}^{H} \mathbf{u}_{2}\right|$.

C. $\mathbf{u}_{1}$ and $\mathbf{u}_{2}$ are orthogonal

Proposition 3. If $\mathbf{u}_{1}$ and $\mathbf{u}_{2}$ are electrically orthogonal, the non-unit-norm version of $\mathbf{f}_{\mathrm{RSV}}$ is given as

$$
\mathbf{f}_{\mathrm{RSV}}=\beta_{\mathrm{opt}} \mathbf{v}_{1}+e^{-j \angle \mathbf{v}_{1}^{H} \mathbf{v}_{2}} \sqrt{1-\beta_{\mathrm{opt}}^{2}} \mathbf{v}_{2}
$$




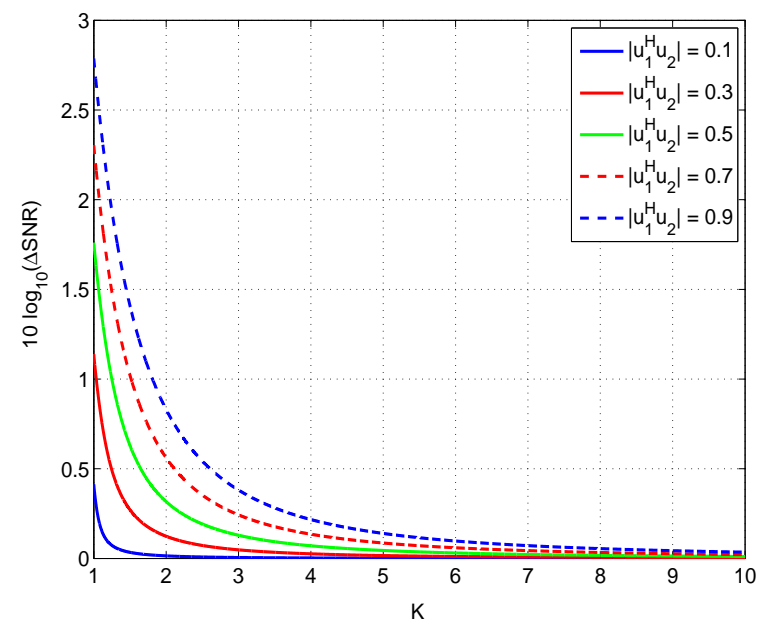

(a)

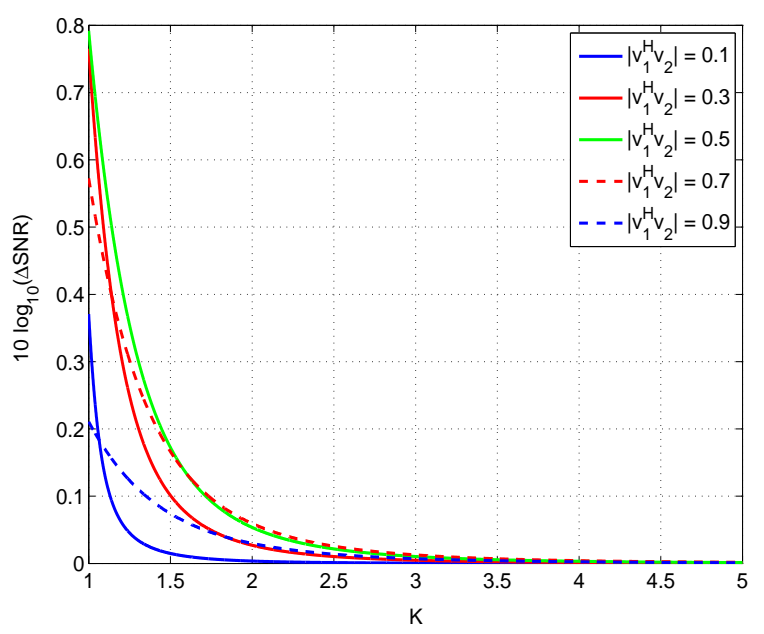

(b)

Fig. 2. $\triangle$ SNR between the optimal beamforming scheme and beamforming along the strongest path as a function of $K$ : (a) as given by (12) when $\mathbf{v}_{1}$ and $\mathbf{v}_{2}$ are orthogonal, and (b) as given by (14) when $\mathbf{u}_{1}$ and $\mathbf{u}_{2}$ are orthogonal.

where

$$
\begin{aligned}
\beta_{\mathrm{opt}}^{2} & =\left\{\begin{array}{cc}
\frac{\mathcal{A}+\sqrt{\mathcal{B}}}{2 \mathcal{C}} & \text { if }\left|\alpha_{1}\right| \geq\left|\alpha_{2}\right| \\
\frac{\mathcal{A}-\sqrt{\mathcal{B}}}{2 \mathcal{C}} & \text { if }\left|\alpha_{1}\right|<\left|\alpha_{2}\right|
\end{array}\right. \text { with } \\
\mathcal{A} & =\frac{\left(\left|\alpha_{1}\right|^{2}-\left|\alpha_{2}\right|^{2}\right)^{2}}{\left|\mathbf{v}_{1}^{H} \mathbf{v}_{2}\right|^{2}}+2\left|\alpha_{1}\right|^{2} \cdot\left(\left|\alpha_{1}\right|^{2}+\left|\alpha_{2}\right|^{2}\right) \\
\mathcal{B} & =\frac{\left(\left|\alpha_{1}\right|^{2}-\left|\alpha_{2}\right|^{2}\right)^{4}}{\left|\mathbf{v}_{1}^{H} \mathbf{v}_{2}\right|^{4}}+\frac{4\left|\alpha_{1}\right|^{2}\left|\alpha_{2}\right|^{2}}{\left|\mathbf{v}_{1}^{H} \mathbf{v}_{2}\right|^{2}} \cdot\left(\left|\alpha_{1}\right|^{2}-\left|\alpha_{2}\right|^{2}\right)^{2} \\
\mathcal{C} & =\left(1+\frac{1}{\left|\mathbf{v}_{1}^{H} \mathbf{v}_{2}\right|^{2}}\right) \cdot\left(\left|\alpha_{1}\right|^{2}+\left|\alpha_{2}\right|^{2}\right)^{2}-\frac{4\left|\alpha_{1}\right|^{2}\left|\alpha_{2}\right|^{2}}{\left|\mathbf{v}_{1}^{H} \mathbf{v}_{2}\right|^{2}} .
\end{aligned}
$$

The non-unit-norm version of $\mathrm{g}_{\mathrm{opt}}$ satisfies

$$
\mathbf{g}_{\mathrm{opt}}=\alpha_{1}\left(\beta_{\mathrm{opt}}+\left|\mathbf{v}_{1}^{H} \mathbf{v}_{2}\right| \sqrt{1-\beta_{\mathrm{opt}}^{2}}\right) \cdot \mathbf{u}_{1} .
$$

\section{Proof. See Appendix C.}

Fig. 1(b) plots $\beta_{\mathrm{opt}}^{2}$ as a function of $\left|\mathbf{v}_{1}^{H} \mathbf{v}_{2}\right|$ for different choices of $K=\frac{\left|\alpha_{1}\right|}{\left|\alpha_{2}\right|}$. As before, Fig. 1 (b) shows that $\beta_{\mathrm{opt}}$ converges to 0 or 1 as $\mathbf{v}_{1}$ and $\mathbf{v}_{2}$ become more orthogonal. A straightforward calculation also shows that

$$
\left|\mathbf{v}_{1}^{H} \mathbf{v}_{2}\right| \rightarrow 1 \Longrightarrow \beta_{\mathrm{opt}}^{2} \rightarrow \frac{\left|\alpha_{1}\right|^{4}}{\left|\alpha_{1}\right|^{4}+\left|\alpha_{2}\right|^{4}}
$$


In between these two extremes, we have

$$
\frac{1}{L} \cdot \max \left(\left|\alpha_{1}\right|^{2},\left|\alpha_{2}\right|^{2}\right) \leq \widetilde{\operatorname{SNR}}_{\mathrm{rx}} \leq \frac{\left|\alpha_{1}\right|^{2}+\left|\alpha_{2}\right|^{2}}{L}
$$

In terms of loss with respect to beamforming along the dominant path, a simple calculation shows that

$$
\Delta \mathrm{SNR}=\frac{\left.\widetilde{\mathrm{SNR}}_{\mathrm{rr}}\right|_{\mathrm{RSV}}}{\left.\widetilde{\mathrm{SNR}_{\mathrm{rx}}}\right|_{\text {Dom. path }}}=\frac{\left|\alpha_{1}\right|^{2}+\left|\alpha_{2}\right|^{2}-\left(1-\left|\mathbf{v}_{1}^{H} \mathbf{v}_{2}\right|^{2}\right) \cdot\left(\frac{\beta_{\mathrm{opt}}^{2} \cdot\left|\alpha_{2}\right|^{2}+\left(1-\beta_{\mathrm{opt}}^{2}\right) \cdot\left|\alpha_{1}\right|^{2}}{1+2 \beta_{\mathrm{opt}} \sqrt{1-\beta_{\mathrm{opt}}^{2}} \cdot\left|\mathbf{v}_{1}^{H} \mathbf{v}_{2}\right|}\right)}{\max \left(\left|\alpha_{1}\right|^{2}+\left|\alpha_{2}\right|^{2}\left|\mathbf{v}_{1}^{H} \mathbf{v}_{2}\right|^{2},\left|\alpha_{2}\right|^{2}+\left|\alpha_{1}\right|^{2}\left|\mathbf{v}_{1}^{H} \mathbf{v}_{2}\right|^{2}\right)}
$$

where $\beta_{\mathrm{opt}}$ is as in the statement of the proposition. While this expression is also hard to visualize, Fig. 2(b) plots it as a function of $K=\frac{\left|\alpha_{1}\right|}{\left|\alpha_{2}\right|}$ for different values of $\left|\mathbf{v}_{1}^{H} \mathbf{v}_{2}\right|$. With $K=\frac{\left|\alpha_{1}\right|}{\left|\alpha_{2}\right|} \geq 1$, note that $\triangle$ SNR can be rewritten as

$$
\Delta \mathrm{SNR}=1+\left[\frac{\left(1-\left|\mathbf{v}_{1}^{H} \mathbf{v}_{2}\right|^{2}\right) \cdot \sqrt{1-\beta_{\mathrm{opt}}^{2}}}{1+2 \beta_{\mathrm{opt}} \sqrt{1-\beta_{\mathrm{opt}}^{2}} \cdot\left|\mathbf{v}_{1}^{H} \mathbf{v}_{2}\right|}\right] \cdot\left[\frac{\sqrt{1-\beta_{\mathrm{opt}}^{2}} \cdot\left(1-K^{2}\right)+2 \beta_{\mathrm{opt}}\left|\mathbf{v}_{1}^{H} \mathbf{v}_{2}\right|}{K^{2}+\left|\mathbf{v}_{1}^{H} \mathbf{v}_{2}\right|^{2}}\right] .
$$

While optimizing the above expression in terms of $K$ is difficult given the complicated functional involvement of $K$ in the above expression, by treating $\beta_{\mathrm{opt}}$ as a fixed quantity, it is straightforward to see that the above expression is decreasing in $K$. Without being rigorous, this argument suggests that the above expression is maximized at $K=1$. Substituting $K=1$, we have $\beta_{\mathrm{opt}}^{2}=\frac{1}{2}$ and

$$
\Delta \mathrm{SNR}=\frac{1+\left|\mathbf{v}_{1}^{H} \mathbf{v}_{2}\right|}{1+\left|\mathbf{v}_{1}^{H} \mathbf{v}_{2}\right|^{2}}
$$

It is easy to see that the above expression is maximized at $\left|\mathbf{v}_{1}^{H} \mathbf{v}_{2}\right|=\sqrt{2}-1$ with a maximum value of $\triangle \mathrm{SNR}=\frac{\sqrt{2}+1}{2}=0.8175 \mathrm{~dB}$. Thus, beamforming along the dominant path is no worser than $0.8175 \mathrm{~dB}$ in terms of optimal beamforming performance. This trend is reinforced by the $\triangle$ SNR plot in Fig. 2(b) as a function of $K=\frac{\left|\alpha_{1}\right|}{\left|\alpha_{2}\right|}$ for different values of $\left|\mathbf{v}_{1}^{H} \mathbf{v}_{2}\right|$.

\section{D. $\mathbf{v}_{1}$ and $\mathbf{v}_{2}$ are parallel}

If $\mathbf{v}_{1}$ and $\mathbf{v}_{2}$ are parallel (or nearly parallel), we can use $\left|\mathbf{v}_{1}^{H} \mathbf{v}_{2}\right| \approx 1$ to rewrite $\widetilde{S N R}_{r x}$ as

$$
\begin{aligned}
\widetilde{\operatorname{SNR}}_{\mathrm{rx}} & =\frac{\left|\alpha_{1}\right|^{2}+\left|\alpha_{2}\right|^{2}+2\left|\alpha_{1}\right|\left|\alpha_{2}\right| \cdot\left|\mathbf{u}_{1}^{H} \mathbf{u}_{2}\right| \cdot \cos (\nu)+2 \beta \sqrt{1-\beta^{2}} \cdot \cos (\phi) \cdot}{\left(\left|\alpha_{1}\right|^{2}+\left|\alpha_{2}\right|^{2}+2\left|\alpha_{1}\right|\left|\alpha_{2}\right| \cdot\left|\mathbf{u}_{1}^{H} \mathbf{u}_{2}\right| \cdot \cos (\nu)\right)} \\
L \cdot\left(1+2 \beta \sqrt{1-\beta^{2}} \cdot \cos (\phi)\right) & \frac{1}{L} \cdot\left(\left|\alpha_{1}\right|^{2}+\left|\alpha_{2}\right|^{2}+2\left|\alpha_{1}\right|\left|\alpha_{2}\right| \cdot\left|\mathbf{u}_{1}^{H} \mathbf{u}_{2}\right| \cdot \cos (\nu)\right) .
\end{aligned}
$$


Clearly, the above objective function is independent of $\beta$ and $\theta$. Therefore, any power allocation scheme across the two paths achieves the above gain. A corollary of this observation is that beamforming along the dominant path is as good as the optimal beamforming scheme $(\Delta \mathrm{SNR}=$ 1).

E. $\mathbf{u}_{1}$ and $\mathbf{u}_{2}$ are parallel

Proposition 4. If $\mathbf{u}_{1}$ and $\mathbf{u}_{2}$ are parallel, the non-unit-norm version of $\mathbf{f}_{\mathrm{RSV}}$ is given as

$$
\mathbf{f}_{\mathrm{RSV}}=\beta_{\mathrm{opt}} \mathbf{v}_{1}+e^{j\left(\angle \alpha_{1}-\angle \alpha_{2}-\angle \mathbf{u}_{1}^{H} \mathbf{u}_{2}\right)} \sqrt{1-\beta_{\mathrm{opt}}^{2}} \mathbf{v}_{2}
$$

where

$$
\beta_{\mathrm{opt}}^{2}=\frac{\left|\alpha_{1}\right|^{2}}{\left|\alpha_{1}\right|^{2}+\left|\alpha_{2}\right|^{2}} .
$$

The non-unit-norm version of $\mathrm{g}_{\mathrm{opt}}$ follows from expanding out $\mathrm{Hf}_{\mathrm{opt}}$ and is not provided here.

Proof. See Appendix D.

Note that $\beta_{\mathrm{opt}}^{2}$ mimics a maximum ratio combining solution, allocating power to each path in proportion to the gain of that path. With $K=\frac{\left|\alpha_{1}\right|}{\left|\alpha_{2}\right|} \geq 1$, the SNR loss can be written as

$$
\begin{aligned}
\Delta \mathrm{SNR} & =\frac{\left.\widetilde{\mathrm{SNR}_{\mathrm{rx}}}\right|_{\mathrm{RSV}}}{\left.\widetilde{\mathrm{SNR}}_{\mathrm{rx}}\right|_{\text {Dom. path }}} \\
& =\frac{\left|\alpha_{1}\right|^{2}+\left|\alpha_{2}\right|^{2}+2\left|\alpha_{1}\right|\left|\alpha_{2}\right| \cos (\nu) \cdot\left|\mathbf{v}_{1}^{H} \mathbf{v}_{2}\right|}{\max \left(\left|\alpha_{1}\right|^{2}+\left|\mathbf{v}_{1}^{H} \mathbf{v}_{2}\right|^{2}\left|\alpha_{2}\right|^{2},\left|\alpha_{2}\right|^{2}+\left|\mathbf{v}_{1}^{H} \mathbf{v}_{2}\right|^{2}\left|\alpha_{1}\right|^{2}\right)+2\left|\alpha_{1}\right|\left|\alpha_{2}\right|\left|\mathbf{v}_{1}^{H} \mathbf{v}_{2}\right| \cos (\nu)} \\
& =1+\frac{\left|\alpha_{2}\right|^{2} \cdot\left(1-\left|\mathbf{v}_{1}^{H} \mathbf{v}_{2}\right|^{2}\right)}{\left|\alpha_{1}\right|^{2}+\left|\mathbf{v}_{1}^{H} \mathbf{v}_{2}\right|^{2}\left|\alpha_{2}\right|^{2}+2\left|\alpha_{1}\right|\left|\alpha_{2}\right|\left|\mathbf{v}_{1}^{H} \mathbf{v}_{2}\right| \cdot \cos (\nu)}
\end{aligned}
$$

This SNR loss term is plotted in Fig. 3 as a function of $K=\frac{\left|\alpha_{1}\right|}{\left|\alpha_{2}\right|}$ for different choices of $\left|\mathbf{v}_{1}^{H} \mathbf{v}_{2}\right|$ and $\nu$. From this study, we see that $\triangle$ SNR can be significantly larger than $3 \mathrm{~dB}$ provided that both paths are approximately similar in terms of gain and are also essentially parallel, but with opposite phases (characterized by $\nu=180^{\circ}$ ). In this setting, the right singular vector combines the gains in both paths by appropriate phase compensation. On the other hand, beamforming along only the strongest path leads to destructive interference of the signal from the sub-dominant path resulting in significant performance loss. Barring these extreme conditions, this study also shows that the performance loss is similar to the $3 \mathrm{~dB}$ characterization in other settings. 


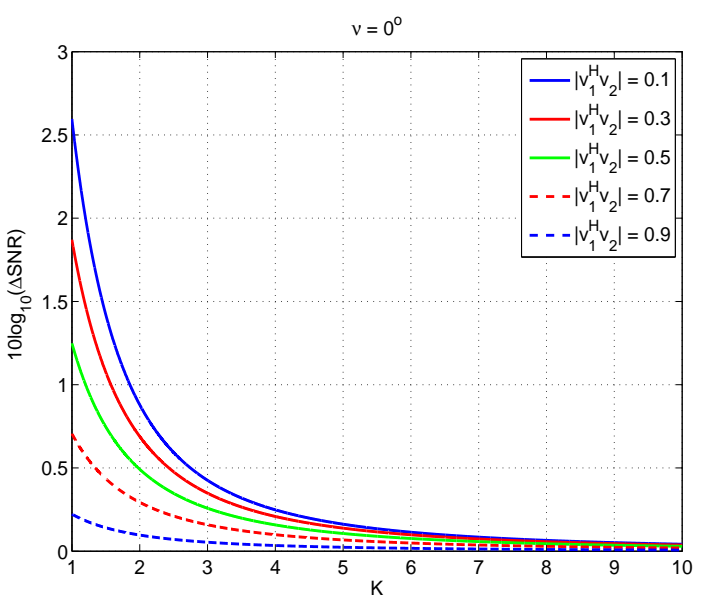

(a)

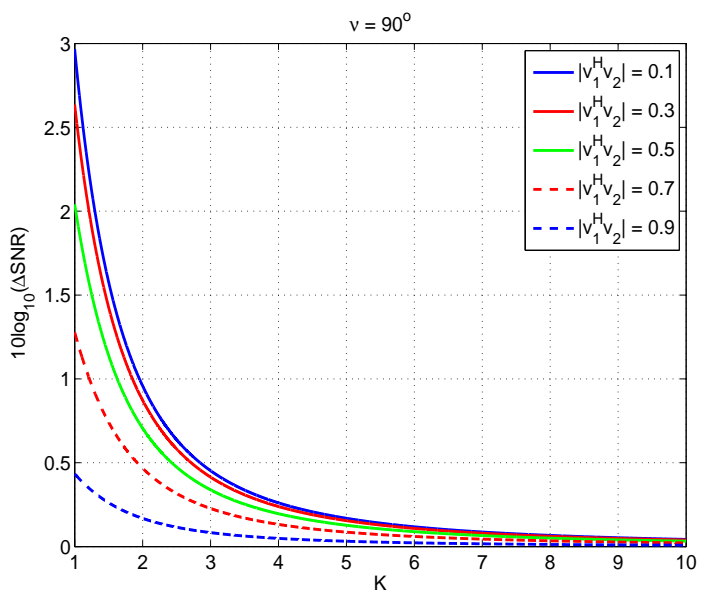

(c)

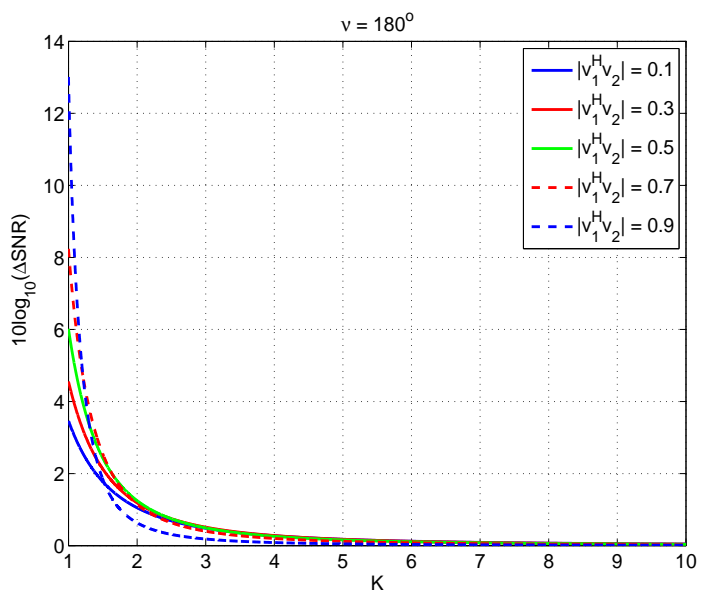

(e)

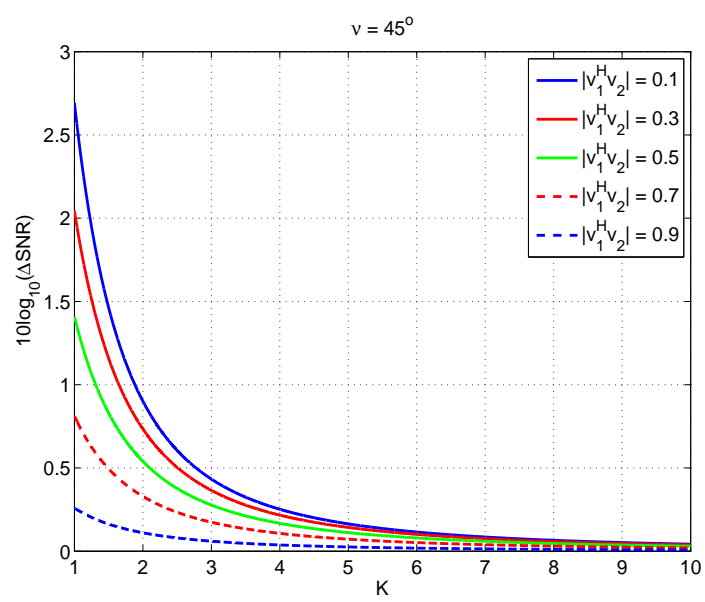

(b)

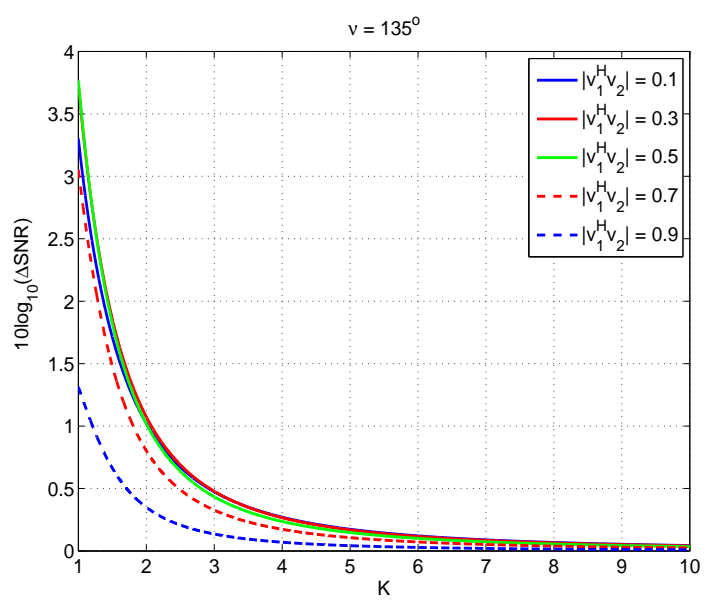

(d)

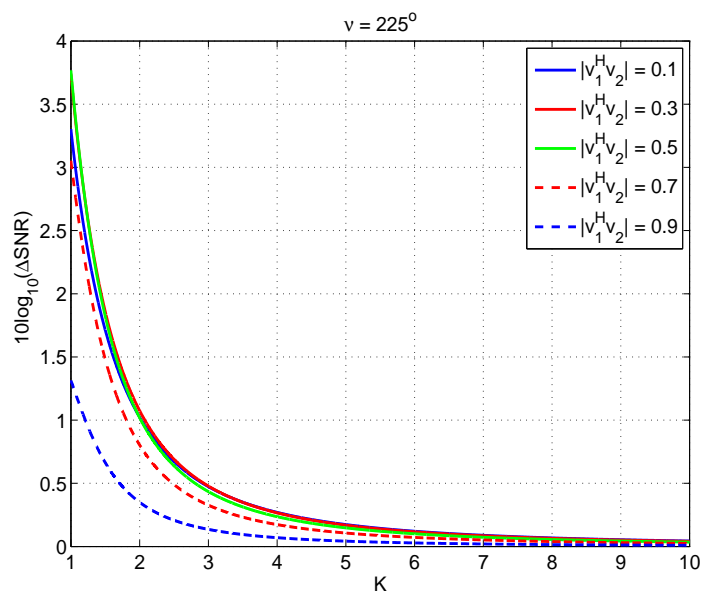

(f)

Fig. 3. $\triangle$ SNR between the optimal beamforming scheme and beamforming along the strongest path as a function of $K$ when $\mathbf{u}_{1}$ and $\mathbf{u}_{2}$ are parallel for different choices of $\nu$ : (a) $\nu=0^{\circ}$, (b) $\nu=45^{\circ}$, (c) $\nu=90^{\circ}$, (d) $\nu=135^{\circ}$, (e) $\nu=180^{\circ}$, and (f) $\nu=225^{\circ}$. 


\section{F. Beamforming with equal power allocation}

Another simple scheme allocates power equally to both the directions $\left(\beta=\frac{1}{\sqrt{2}}\right)$. Note that this scheme requires a digital beamformer (in general) since the sum of two CPO beams does not have a constant amplitude. For this scheme, it is straightforward to see that

$$
\begin{aligned}
\widetilde{\mathrm{SNR}}_{\mathrm{rx}} & =\frac{\mathcal{A}_{1}+\mathcal{A}_{2}}{L \cdot 2\left(1+\left|\mathbf{v}_{1}^{H} \mathbf{v}_{2}\right| \cdot \cos (\phi)\right)} \\
\mathcal{A}_{1} & =\left(\left|\alpha_{1}\right|^{2}+\left|\alpha_{2}\right|^{2}\right) \cdot\left[1+\left|\mathbf{v}_{1}^{H} \mathbf{v}_{2}\right|^{2}+2\left|\mathbf{v}_{1}^{H} \mathbf{v}_{2}\right| \cos (\phi)\right] \\
\mathcal{A}_{2} & =2\left|\alpha_{1}\right|\left|\alpha_{2}\right| \cdot\left|\mathbf{u}_{1}^{H} \mathbf{u}_{2}\right| \cdot\left[\left|\mathbf{v}_{1}^{H} \mathbf{v}_{2}\right|^{2} \cdot \cos (\nu+\phi)+2\left|\mathbf{v}_{1}^{H} \mathbf{v}_{2}\right| \cdot \cos (\nu)+\cos (\nu-\phi)\right] .
\end{aligned}
$$

While the optimal choice of $\phi$ is unclear for this scheme, in the scenario of coherent phase alignment $(\phi=\nu=0)$, with $\max \left(\left|\alpha_{1}\right|^{2},\left|\alpha_{2}\right|^{2}\right)=K^{2}$ and $\min \left(\left|\alpha_{1}\right|^{2},\left|\alpha_{2}\right|^{2}\right)=1$ where $K \geq 1$, we have

$$
\begin{aligned}
\widetilde{\mathrm{SNR}}_{\mathrm{rx}} & =\frac{\left(\left|\alpha_{1}\right|^{2}+\left|\alpha_{2}\right|^{2}+2\left|\alpha_{1}\right|\left|\alpha_{2}\right| \cdot\left|\mathbf{u}_{1}^{H} \mathbf{u}_{2}\right|\right) \cdot\left(1+\left|\mathbf{v}_{1}^{H} \mathbf{v}_{2}\right|\right)^{2}}{L \cdot 2\left(1+\left|\mathbf{v}_{1}^{H} \mathbf{v}_{2}\right|\right)} \\
& =\frac{\left(1+\left|\mathbf{v}_{1}^{H} \mathbf{v}_{2}\right|\right)}{L \cdot 2} \cdot\left(\left|\alpha_{1}\right|^{2}+\left|\alpha_{2}\right|^{2}+2\left|\alpha_{1}\right|\left|\alpha_{2}\right| \cdot\left|\mathbf{u}_{1}^{H} \mathbf{u}_{2}\right|\right) \\
& =\frac{\left(1+\left|\mathbf{v}_{1}^{H} \mathbf{v}_{2}\right|\right)}{L \cdot 2} \cdot\left(K^{2}+1+2 K \cdot\left|\mathbf{u}_{1}^{H} \mathbf{u}_{2}\right|\right)
\end{aligned}
$$

Under favorable channel conditions $\left(\left\{\left|\mathbf{v}_{1}^{H} \mathbf{v}_{2}\right|,\left|\mathbf{u}_{1}^{H} \mathbf{u}_{2}\right|\right\} \approx 1\right)$, equal power beamforming can add signals coherently to yield $\frac{(K+1)^{2}}{L}$, whereas when $K \gg 1$, we have a gain of $\frac{K^{2}}{L} \cdot \frac{1+\left|\mathbf{v}_{1}^{H} \mathbf{v}_{2}\right|}{2}$. If $\mathbf{v}_{1}$ and $\mathbf{v}_{2}$ ar electrically orthogonal, it is clear that half the power (along $\mathbf{v}_{2}$ ) is wasted resulting in a $3 \mathrm{~dB}$ loss over the scheme where the entire power is directed along the dominant path $\left(\mathbf{v}_{1}\right)$.

\section{Directional Beamforming at Both Ends}

While we have so far considered the case of directional beamforming at the transmitter, the receiver uses a matched filter corresponding to such a scheme, which may not be directional. We now consider the case of directional beamforming at both ends. In Fig. 4, we plot the complementary cumulative distribution function $(\mathrm{CCDF})$ of the loss in $\mathrm{SNR}_{\mathrm{rx}}$ with such a bidirectional scheme relative to the optimal beamforming scheme for different choices of $L$. The gains of the paths as well as their directions are chosen independently and identically distributed (i.i.d.) from a certain path loss model and over the $120^{\circ}$ field-of-view of the arrays. From this figure, we note that for a large fraction of the channel realizations, beamforming along the 


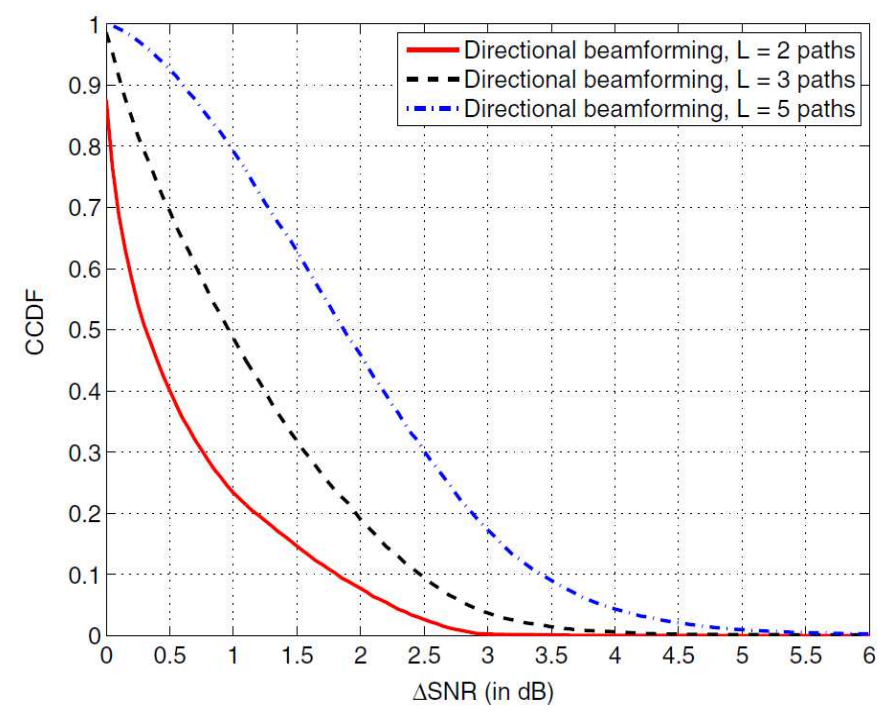

Fig. 4. Complementary CDF of $\triangle \mathrm{SNR}$ as a function of $L$.

dominant direction only results in a small performance loss. In particular, the median losses in the three cases $(L=2,3$ and 5 ) are $0.3 \mathrm{~dB}, 0.95 \mathrm{~dB}$ and $1.85 \mathrm{~dB}$, and the 90-th percentile losses are $1.8 \mathrm{~dB}, 2.45 \mathrm{~dB}$ and $3.4 \mathrm{~dB}$. Thus, this study suggests that directional beamforming could serve as a useful low-complexity scheme with good performance in the mmW regime.

\section{CONCLUDing REMARKS}

This paper developed an explicit mapping and dependence of the optimal beamformer structure on the different aspects of the sparse channel that characterize propagation in the $\mathrm{mmW}$ regime. This study showed that the optimal beamformer approaches dominant path (directional) beamforming as either the AoDs or AoAs of the paths become more (electrically) orthogonal. In general, if the AoDs or AoAs are not orthogonal, optimal beamforming entails appropriate power allocation and phase compensation across the paths. While specific channel realizations can be constructed to ensure that directional beamforming can suffer significantly relative to the optimal scheme, in a distributional sense, the loss in received SNR is expected to be minimal. Furthermore, this small additional gain in received SNR with optimal beamforming comes at the cost of tight phase synchronization across paths, an onerous task at $\mathrm{mmW}$ frequencies especially since relative motion on the order of the wavelength (a few millimeters) can render the 
optimal beamformer unuseable in practice. These conclusions on small losses with directional beamforming as well as its robustness relative to the optimal scheme provides a major fillip to the search for good directional learning approaches, a task that has received significant and increasing attention in the literature.

\section{APPENDIX}

\section{A. Proof of Prop. 1}

The $N_{t} \times N_{t}$ matrix $\mathrm{H}^{H} \mathrm{H}$ can be expanded as

$$
\begin{aligned}
\frac{L}{N_{t} N_{r}} \cdot \mathrm{H}^{H} \mathbf{H} & =\sum_{i, j} \alpha_{i}^{\star} \alpha_{j} \cdot\left(\mathbf{u}_{i}^{H} \mathbf{u}_{j}\right) \cdot \mathbf{v}_{i} \mathbf{v}_{j}^{H} \\
& =\operatorname{VAV}^{H}
\end{aligned}
$$

where $\mathbf{V}=\left[\alpha_{1}^{\star} \mathbf{v}_{1}, \cdots, \alpha_{L}^{\star} \mathbf{v}_{L}\right]$ and $\mathrm{A}(i, j)=\mathbf{u}_{i}^{H} \mathbf{u}_{j}, i, j=1, \cdots, L$. Let $\mathbf{X}$ be an $L \times L$ eigenvector matrix of $A \mathrm{~V}^{H} \mathrm{~V}$ with the corresponding diagonal matrix of eigenvalues denoted by $D$. That is (the eigenvalue equation is given as),

$$
\left(A \vee^{H} \mathrm{~V}\right) \cdot \mathrm{X}=\mathrm{X} \cdot \mathrm{D}
$$

Pre-multiplying both sides of (16) by $\mathrm{V}$, we have

$$
\mathrm{VX} \cdot \mathrm{D}=\left(\mathrm{VAV}^{H} \mathrm{~V}\right) \cdot \mathrm{X}=\left(\frac{L}{N_{t} N_{r}} \cdot \mathrm{H}^{H} \mathrm{H}\right) \cdot \mathrm{VX}
$$

Reading equation (17) from right to left, we see that $\mathrm{VX}$ forms the eigenvector matrix for $\mathrm{H}^{H} \mathrm{H}$ with the diagonal eigenvalue matrix being the same as D. In other words, all the eigenvectors of $\mathrm{H}^{H} \mathrm{H}$ can be represented as linear combinations of $\mathbf{v}_{1}, \cdots, \mathbf{v}_{L}$. The only difference between the $L \leq N_{t}$ and $L>N_{t}$ cases is that the number of distinct eigenvectors of $\mathrm{X}$ is less than or equal to $L$ and $N_{t}$ in the two cases, respectively.

Given the structure of $\mathbf{f}_{\mathrm{opt}}=\sum_{j=1}^{L} \beta_{j} \mathbf{v}_{j}$, we have

$$
\begin{aligned}
\mathbf{H f}_{\mathrm{opt}} & =\left(\sum_{i=1}^{L} \alpha_{i} \mathbf{u}_{i} \mathbf{v}_{i}^{H}\right) \cdot\left(\sum_{j=1}^{L} \beta_{j} \mathbf{v}_{j}\right) \\
& =\sum_{i=1}^{L} \alpha_{i} \cdot\left(\sum_{j} \beta_{j} \mathbf{v}_{i}^{H} \mathbf{v}_{j}\right) \mathbf{u}_{i}
\end{aligned}
$$

and thus $\mathbf{g}_{\text {opt }}$ is a linear combination of $\left\{\mathbf{u}_{1}, \cdots, \mathbf{u}_{L}\right\}$. 


\section{B. Proof of Prop. 2}

A simple substitution of $\mathbf{v}_{1}^{H} \mathbf{v}_{2}=0$ leads to

$$
\widetilde{\operatorname{SNR}}_{\mathrm{rx}}=\frac{1}{L} \cdot\left[\beta^{2}\left|\alpha_{1}\right|^{2}+\left(1-\beta^{2}\right)\left|\alpha_{2}\right|^{2}+2 \beta \sqrt{1-\beta^{2}} \cdot\left|\alpha_{1}\right|\left|\alpha_{2}\right| \cdot\left|\mathbf{u}_{1}^{H} \mathbf{u}_{2}\right| \cdot \cos (\nu-\phi)\right],
$$

which upon optimization over $\phi$ results in

$$
\widetilde{\operatorname{SNR}}_{\mathrm{rx}}=\frac{1}{L} \cdot\left[\beta^{2}\left|\alpha_{1}\right|^{2}+\left(1-\beta^{2}\right)\left|\alpha_{2}\right|^{2}+2 \beta \sqrt{1-\beta^{2}} \cdot\left|\alpha_{1}\right|\left|\alpha_{2}\right| \cdot\left|\mathbf{u}_{1}^{H} \mathbf{u}_{2}\right|\right] .
$$

A straightforward computation shows that the optimal solution to the above optimization in the $\beta$ variable satisfies the quadratic equation

$$
\beta^{2}\left(1-\beta^{2}\right) \cdot\left(\left|\alpha_{1}\right|^{2}-\left|\alpha_{2}\right|^{2}\right)^{2}=\left|\alpha_{1}\right|^{2}\left|\alpha_{2}\right|^{2}\left|\mathbf{u}_{1}^{H} \mathbf{u}_{2}\right|^{2} \cdot\left(2 \beta^{2}-1\right)^{2}
$$

and is of the form in (10). A straightforward substitution of the structure of $f_{o p t}$ in (19) results in $\mathrm{g}_{\mathrm{opt}}$.

\section{Proof of Prop. 3}

When $\mathbf{u}_{1}$ and $\mathbf{u}_{2}$ are orthogonal, we have

$$
\begin{aligned}
\widetilde{\mathrm{SNR}}_{\mathrm{rx}} & =\frac{A \cdot\left(\left|\alpha_{1}\right|^{2}+\left|\alpha_{2}\right|^{2}\right)+2\left(\left|\alpha_{1}\right|^{2}+\left|\alpha_{2}\right|^{2}\right) \cdot \beta \sqrt{1-\beta^{2}} \cdot\left|\mathbf{v}_{1}^{H} \mathbf{v}_{2}\right| \cdot \cos (\phi)}{L \cdot\left(1+2 \beta \sqrt{1-\beta^{2}}\left|\mathbf{v}_{1}^{H} \mathbf{v}_{2}\right| \cos (\phi)\right)} \\
& =\left(\frac{\left|\alpha_{1}\right|^{2}+\left|\alpha_{2}\right|^{2}}{L}\right) \cdot \frac{A+2 \beta \sqrt{1-\beta^{2}} \cdot\left|\mathbf{v}_{1}^{H} \mathbf{v}_{2}\right| \cdot \cos (\phi)}{1+2 \beta \sqrt{1-\beta^{2}} \cdot\left|\mathbf{v}_{1}^{H} \mathbf{v}_{2}\right| \cdot \cos (\phi)}
\end{aligned}
$$

where

$$
A=\frac{\beta^{2}\left(\left|\alpha_{1}\right|^{2}+\left|\alpha_{2}\right|^{2}\left|\mathbf{v}_{1}^{H} \mathbf{v}_{2}\right|^{2}\right)+\left(1-\beta^{2}\right)\left(\left|\alpha_{2}\right|^{2}+\left|\alpha_{1}\right|^{2}\left|\mathbf{v}_{1}^{H} \mathbf{v}_{2}\right|^{2}\right)}{\left|\alpha_{1}\right|^{2}+\left|\alpha_{2}\right|^{2}} \leq 1 .
$$

Since $A \leq 1$ for all choices of $\beta^{2}$, it is easy to see that $\widetilde{\operatorname{SNR}}_{\mathrm{rx}}$ is always maximized when $\cos (\phi)$ is maximized at 1 by the choice $\theta=-\angle \mathbf{v}_{1}^{H} \mathbf{v}_{2}$. Using this fact, after some manipulations, we have the following:

$$
\begin{aligned}
\widetilde{\mathrm{SNR}}_{\mathrm{rx}} & =\frac{\left|\alpha_{1}\right|^{2} \cdot\left(\beta+\sqrt{1-\beta^{2}}\left|\mathbf{v}_{1}^{H} \mathbf{v}_{2}\right|\right)^{2}+\left|\alpha_{2}\right|^{2} \cdot\left(\beta\left|\mathbf{v}_{1}^{H} \mathbf{v}_{2}\right|+\sqrt{1-\beta^{2}}\right)^{2}}{L \cdot\left(1+2 \beta \sqrt{1-\beta^{2}}\left|\mathbf{v}_{1}^{H} \mathbf{v}_{2}\right|\right)} \\
& =\frac{\left|\alpha_{1}\right|^{2}+\left|\alpha_{2}\right|^{2}}{L}-\left(\frac{1-\left|\mathbf{v}_{1}^{H} \mathbf{v}_{2}\right|^{2}}{L}\right) \cdot\left(\frac{\beta^{2} \cdot\left|\alpha_{2}\right|^{2}+\left(1-\beta^{2}\right) \cdot\left|\alpha_{1}\right|^{2}}{1+2 \beta \sqrt{1-\beta^{2}} \cdot\left|\mathbf{v}_{1}^{H} \mathbf{v}_{2}\right|}\right)
\end{aligned}
$$


Thus, the optimal choice of $\beta^{2}$ (denoted as $\beta_{\mathrm{opt}}^{2}$ ) is that choice that minimizes the quantity in the parentheses above. It can be seen that this optimal choice satisfies the equation:

$$
\beta_{\mathrm{opt}}^{2} \cdot\left|\alpha_{2}\right|^{2}-\left(1-\beta_{\mathrm{opt}}^{2}\right) \cdot\left|\alpha_{1}\right|^{2}=\beta_{\mathrm{opt}} \cdot \sqrt{1-\beta_{\mathrm{opt}}^{2}} \cdot \frac{\left(\left|\alpha_{1}\right|^{2}-\left|\alpha_{2}\right|^{2}\right)}{\left|\mathbf{v}_{1}^{H} \mathbf{v}_{2}\right|}
$$

and is explicitly written as in the statement of the proposition. A straightforward substitution of $\mathrm{f}_{\text {opt }}$ in (19) results in $\mathrm{g}_{\mathrm{opt}}$.

D. Proof of Prop. 4

In this setting, we can use the fact that $\left|\mathbf{u}_{1}^{H} \mathbf{u}_{2}\right| \approx 1$ to rewrite $\widetilde{\mathrm{SNR}}_{\mathrm{rx}}$ as

$$
\begin{aligned}
\widetilde{\mathrm{SNR}}_{\mathrm{rx}} & =\frac{\left|\alpha_{1}\right|^{2}+\left|\alpha_{2}\right|^{2}+\frac{2\left|\alpha_{1}\right|\left|\alpha_{2}\right| \cos (\nu)}{\left|\mathbf{v}_{1}^{H} \mathbf{v}_{2}\right|}}{L}-\frac{\left(1-\left|\mathbf{v}_{1}^{H} \mathbf{v}_{2}\right|^{2}\right)}{L} \cdot f(\beta, \phi) \\
f(\beta, \phi) & \triangleq \frac{\left|\alpha_{1}\right|^{2} \cdot\left(1-\beta^{2}\right)+\left|\alpha_{2}\right|^{2} \cdot \beta^{2}+\frac{2\left|\alpha_{1}\right|\left|\alpha_{2}\right| \cos (\nu)}{\left|\mathbf{v}_{1}^{H} \mathbf{v}_{2}\right|}+2 \beta \sqrt{1-\beta^{2}} \cdot\left|\alpha_{1}\right|\left|\alpha_{2}\right| \cdot \cos (\nu+\phi)}{1+2 \beta \sqrt{1-\beta^{2}} \cdot\left|\mathbf{v}_{1}^{H} \mathbf{v}_{2}\right| \cdot \cos (\phi)} .
\end{aligned}
$$

To find the structure of $\mathbf{f}_{\mathrm{RSV}}$, we need to find $\left\{\beta^{\star}, \phi^{\star}\right\}=\arg \min _{\beta, \phi} f(\beta, \phi)$.

We now claim that for any $\nu, \beta, K=\frac{\left|\alpha_{1}\right|}{\left|\alpha_{2}\right|} \geq 1, \phi^{\star}=\nu$. Substituting $\phi^{\star}=\nu$, we have

$$
\begin{aligned}
& f\left(\beta, \phi^{\star}\right)= \frac{\left|\alpha_{1}\right|^{2} \cdot\left(1-\beta^{2}\right)+\left|\alpha_{2}\right|^{2} \cdot \beta^{2}+\frac{2\left|\alpha_{1}\right|\left|\alpha_{2}\right| \cos (\nu)}{\left|\mathbf{v}_{1}^{H} \mathbf{v}_{2}\right|}+2 \beta \sqrt{1-\beta^{2}} \cdot\left|\alpha_{1}\right|\left|\alpha_{2}\right| \cdot \cos (2 \nu)}{1+2 \beta \sqrt{1-\beta^{2}} \cdot\left|\mathbf{v}_{1}^{H} \mathbf{v}_{2}\right| \cdot \cos (\nu)} \\
&= \frac{2\left|\alpha_{1}\right|\left|\alpha_{2}\right| \cos (\nu)}{\left|\mathbf{v}_{1}^{H} \mathbf{v}_{2}\right|}+\frac{\left(\left|\alpha_{1}\right| \sqrt{1-\beta^{2}}-\left|\alpha_{2}\right| \beta\right)^{2}}{1+2 \beta \sqrt{1-\beta^{2}} \cdot\left|\mathbf{v}_{1}^{H} \mathbf{v}_{2}\right| \cdot \cos (\nu)} \\
& \widetilde{\operatorname{SNR}}_{\mathrm{rx}}= \frac{\left|\alpha_{1}\right|^{2}+\left|\alpha_{2}\right|^{2}+2\left|\alpha_{1}\right|\left|\alpha_{2}\right| \cos (\nu) \cdot\left|\mathbf{v}_{1}^{H} \mathbf{v}_{2}\right|}{L} \\
&-\frac{\left(1-\left|\mathbf{v}_{1}^{H} \mathbf{v}_{2}\right|^{2}\right)}{L} \cdot \min _{\beta} \frac{\left(\left|\alpha_{1}\right| \sqrt{1-\beta^{2}}-\left|\alpha_{2}\right| \beta\right)^{2}}{1+2 \beta \sqrt{1-\beta^{2}} \cdot\left|\mathbf{v}_{1}^{H} \mathbf{v}_{2}\right| \cos (\nu)} .
\end{aligned}
$$

By setting $\beta^{2}=\beta_{\mathrm{opt}}^{2}$, the last term can be minimized (to 0 ), resulting in:

$$
\widetilde{\operatorname{SNR}}_{\mathrm{rx}}=\frac{\left|\alpha_{1}\right|^{2}+\left|\alpha_{2}\right|^{2}+2\left|\alpha_{1}\right|\left|\alpha_{2}\right| \cos (\nu) \cdot\left|\mathbf{v}_{1}^{H} \mathbf{v}_{2}\right|}{L} .
$$




\section{REFERENCES}

[1] F. Rusek, D. Persson, B. K. Lau, E. G. Larsson, T. L. Marzetta, O. Edfors, and F. Tufvesson, "Scaling up MIMO: Opportunities and challenges with very large arrays," IEEE Sig. Proc. Magaz., vol. 30, no. 1, pp. 40-60, Jan. 2013.

[2] N. Bhushan, J. Li, D. Malladi, R. Gilmore, D. Brenner, A. Damnjanovic, R. T. Sukhasvi, C. Patel, and S. Geirhofer, "Network densification: The dominant theme for wireless evolution into 5G," IEEE Commun. Magaz., vol. 52, no. 2, pp. 82-89, Feb. 2014.

[3] F. Boccardi, F. Tosato, and G. Caire, "Precoding Schemes for the MIMO-GBC," Proc. 2006 Intern. Zurich Seminar on Commun., Zurich, Switzerland, pp. 10-13, Feb. 2006.

[4] Qualcomm, "The 1000-X data challenge," Available: [Online].

http://www. qualcomm.com/solutions/wireless-networks/technologies/1000x-data.

[5] F. Khan and Z. Pi, "An introduction to millimeter wave mobile broadband systems," IEEE Commun. Magaz., vol. 49, no. 6, pp. 101-107, June 2011.

[6] T. S. Rappaport, S. Sun, R. Mayzus, H. Zhao, Y. Azar, K. Wang, G. N. Wong, J. K. Schulz, M. Samimi, and F. Gutierrez, “Millimeter wave mobile communications for 5G cellular: It will work!,” IEEE Access, vol. 1, pp. 335-349, 2013.

[7] S. Rangan, T. S. Rappaport, and E. Erkip, "Millimeter wave cellular networks: Potentials and challenges," Proc. IEEE, vol. 102, no. 3, pp. 366-385, Mar. 2014.

[8] W. Roh, J.-Y. Seol, J. Park, B. Lee, J. Lee, Y. Kim, J. Cho, K. Cheun, and F. Aryanfar, "Millimeter-wave beamforming as an enabling technology for $5 \mathrm{G}$ cellular communications: Theoretical feasibility and prototype results," IEEE Commun. Magaz., vol. 52, no. 2, pp. 106-113, Feb. 2014.

[9] C. Lim, T. Yoo, B. Clerckx, B. Lee, and B. Shim, "Recent trend of multiuser MIMO in LTE-Advanced," IEEE Commun. Magaz., vol. 51, no. 3, pp. 127-135, Mar. 2013.

[10] V. Venkateswaran and A.-J. van der Veen, "Analog beamforming in MIMO communications with phase shift networks and online channel estimation," IEEE Trans. Sig. Proc., vol. 58, no. 8, pp. 4131-4143, Aug. 2010.

[11] E. Torkildson, U. Madhow, and M. Rodwell, "Indoor millimeter wave MIMO: Feasibility and performance," IEEE Trans. Wireless Commun., vol. 10, no. 12, pp. 4150-4160, Dec. 2011.

[12] J. Brady, N. Behdad, and A. M. Sayeed, "Beamspace MIMO for millimeter-wave communications: System architecture, modeling, analysis and measurements," IEEE Trans. Ant. Propag., vol. 61, no. 7, pp. 3814-3827, July 2013.

[13] O. El Ayach, S. Rajagopal, S. Abu-Surra, Z. Pi, and R. W. Heath, Jr., "Spatially sparse precoding in millimeter wave MIMO systems," IEEE Trans. Wireless Commun., vol. 13, no. 3, pp. 1499-1513, Mar. 2014.

[14] S. Hur, T. Kim, D. J. Love, J. V. Krogmeier, T. A. Thomas, and A. Ghosh, "Millimeter wave beamforming for wireless backhaul and access in small cell networks," IEEE Trans. Commun., vol. 61, no. 10, pp. 4391-4403, Oct. 2014.

[15] A. Alkhateeb, O. El Ayach, G. Leus, and R. W. Heath, Jr., "Channel estimation and hybrid precoding for millimeter wave cellular systems,” IEEE Journ. Selected Topics in Sig. Proc., vol. 8, no. 5, pp. 831-846, Oct. 2014.

[16] A. M. Sayeed and V. Raghavan, "Maximizing MIMO capacity in sparse multipath with reconfigurable antenna arrays," IEEE Journ. Selected Topics in Sig. Proc., vol. 1, no. 1, pp. 156-166, June 2007.

[17] V. Raghavan and A. M. Sayeed, "Sublinear capacity scaling laws for sparse MIMO channels," IEEE Trans. Inf. Theory, vol. 57, no. 1, pp. 345-364, Jan. 2011.

[18] G. Hariharan, V. Raghavan, and A. M. Sayeed, "Capacity of sparse wideband channels with partial channel feedback," European Trans. Telecommun., vol. 19, no. 4, pp. 475-493, June 2008. 
[19] A. A. M. Saleh and R. Valenzuela, "A statistical model for indoor multipath propagation," IEEE Journ. Selected Areas in Commun., vol. 5, no. 2, pp. 128-137, Feb. 1987.

[20] C. A. Balanis, Antenna Theory: Analysis and Design, Wiley-Interscience, 3rd edition, 2005.

[21] T. K. Y. Lo, "Maximum ratio transmission," IEEE Trans. Commun., vol. 47, no. 10, pp. 1458-1461, Oct. 1999.

[22] M. Samimi, K. Wang, Y. Azar, G. N. Wong, R. Mayzus, H. Zhao, J. K. Schulz, S. Sun, F. J. Gutierrez, and T. S. Rappaport, "28 GHz angle of arrival and angle of departure analysis for outdoor cellular communications using steerable beam antennas in New York City,” Proc. IEEE Veh. Tech. Conf. (Spring), pp. 1-6, Sept. 2013. 